LBNL-58360

\title{
Review of U.S. Neutrino Factory Studies
}

\author{
M. S. Zisman ${ }^{\dagger}$ \\ Accelerator \& Fusion Research Division, Lawrence Berkeley National Laboratory \\ One Cyclotron Road, Berkeley, CA 94720 USA
}

We summarize the status of the two U.S. feasibility studies carried out by the Neutrino Factory and Muon Collider Collaboration (NFMCC) along with recent improvements to Neutrino Factory design developed during the American Physical Society (APS) Neutrino Physics Study. Suggested accelerator topics for the International Scoping Study (ISS) are also indicated.

\section{INTRODUCTION}

Neutrino Factory design is still evolving worldwide. In the U.S., the design work is carried out by the NFMCC. In Europe, the organizing group is BENE (Beams for European Neutrino Experiments), aided by local groups such as the UK Neutrino Factory Group. Japan also has a Neutrino Working Group to carry out its design effort. This paper reflects the experience gained in the U.S. studies, and should be viewed as representative of what might be needed in future studies, such as the ISS.

The ISS is intended to pin down the ingredients of a Neutrino Factory, and thus will serve to elucidate the issues related to design of such a facility. This study will serve as a prelude to a "World Design Study" that we hope will follow in a few years.

\section{NEUTRINO FACTORY STUDY I}

The first U.S. feasibility study [1], Study I, was carried out during 1999-2000. It was initiated by the Fermilab director, in collaboration with the NFMCC. The study focused on examining the feasibility of a Neutrino Factory. It was the first attempt to specify the facility from end to end. The approach adopted was to base the design mainly on well-understood technologies. No attempt was made to optimize either performance or cost of the facility.

This was unquestionably the proper approach at the time, as the feasibility of building a Neutrino Factory was most at issue then. However, this

\footnotetext{
${ }^{\dagger}$ Work supported by the Director, Office of Science, High Energy Physics, U. S. Department of Energy under Contract No. DE-AC02-05CH11231.
}

approach led to a predictable result - feasibility was established, but the performance of the design was rather poor and the cost was rather high.

It was clear from this initial study that substantially more work was needed to improve the predicted performance of a Neutrino Factory. This became the goal of Study II.

\section{NEUTRINO FACTORY STUDY II}

The second U.S. feasibility study, referred to as Study II, was organized jointly by the NFMCC and Brookhaven National Laboratory [2]. The goal was to maintain the convincing feasibility demonstrated in Study I while improving the performance substantially. Once again, cost optimization was given lower priority. The performance achieved, 1.2 $\times 10^{20}$ electron neutrinos per $10^{7} \mathrm{~s}$ year per MW of incident proton beam, is five times that of Study I. Thus, we succeeded in our primary objective.

The cost of the Study II facility was about $75 \%$ of the cost of the Study I facility, so it might be naively assumed that we succeeded in this regard as well. In reality, however, the decrease in cost was due mainly to the choice of final energy. Study I adopted $50 \mathrm{GeV}$ as the final muon beam energy, whereas Study II adopted $20 \mathrm{GeV}$. The lower energy taken for Study II eliminated one stage of acceleration compared with Study I, and this accounted for the bulk of the savings. Compared at equal final energy, the costs of the two facilities were roughly equal.

\section{LESSONS LEARNED}

In going from Study I to Study II, a number of lessons were learned. The first lesson is that it pays to do "local" optimizations initially, before trying 
more global optimizations of facility design. This teaches the designers about the effects of particular design choices and indicates what compromises and trade-offs are the least harmful to performance.

The second lesson is that we must work as partners with the engineers in order to converge on a buildable design. This is not to say that every detail must be fully engineered from the outset. Rather, a few experienced engineers must serve as consultants to ensure that the concepts proposed are realistic and that the dimensions assumed are realizable. This level of engineering will be required for the ISS.

The third lesson learned is that it is worthwhile to simulate the entire concept - that is, to develop a fully self-consistent solution-before doing detailed engineering. This avoids a traditional problem in design studies of forcing engineers to aim at a "moving target." Ideally, this is the stage of machine design we will reach at the end of the ISS - a fully simulated end-to-end design having acceptable performance and reasonably optimized subsystem costs.

\section{STUDY IIa IMPROVEMENTS}

In Sections 2 and 3, we discussed our studies of Neutrino Factory "design space" that represented low performance and high cost (Study I) and high performance and high cost (Study II). Clearly, the most interesting area of design space-high performance and "optimized" cost-remained to be explored. The opportunity to do so came in the form of the APS Neutrino Physics Study [3]. We took advantage of this activity to explore improvements to the Study II design. Since no engineering help was available for the APS study, the work done was not at a level comparable to that from the earlier Studies I and II. For this reason, we adopted the notation "Study IIa" for this effort, denoting an improvement to Study II, but not enough work to be considered as a next-generation study.

Fortunately, the knowledge gained from the previous studies gave us a good idea of what features needed changing. The items we identified were: i) replace induction linacs with RF bunching and phase rotation; ii) replace the high-energy recirculating linear accelerators (RLAs) with fixedfield alternating-gradient (FFAG) rings; and iii) improve optimization of cooling channel performance vs. acceleration system acceptance.

\subsection{Buncher and Phase Rotation}

In the Study-II design, we employed three induction linacs to phase-rotate the beam, and then an RF channel to bunch it. The system performed quite well, but was rather expensive. In hindsight, another disadvantage was that this scheme limits the facility to transporting only one sign of muons at a time.

In Study IIa, we employ a system where RF is used both to bunch the beam and to phase rotate it. The performance is not quite as good as that of the original concept, but the estimated cost is much lower. On the plus side, such a system transmits both $\mu^{+}$and $\mu^{-}$simultaneously.

A schematic illustration of how the system operates is shown in Fig. 1.

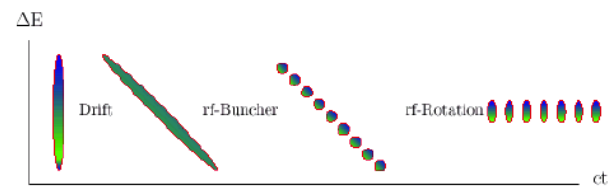

Figure 1. Operation of RF bunching and phase rotation. The beam drifts to establish an energy-time correlation, is bunched in an RF channel having decreasing frequencies, and finally is phase rotated by a second RF channel.

\subsection{Cooling Channel}

The cooling channel was considerably simplified compared with that in Study II. The updated system has fewer magnets, fewer RF cavities, and simpler absorbers. The beam transport (see Fig. 2) is a FOFO system. Solid LiH absorbers are used in place of the liquid-hydrogen system from Study II. Dualpurpose absorbers are combined with the Be windows of the RF cavities as indicated in Fig. 2. This works well because the beta function is nearly constant, making it practical to situate the absorber anywhere we want, as opposed to putting it at the optical focus (i.e., the low-beta point in the lattice).

\subsection{Acceleration}

The updated plan is to use non-scaling FFAG rings

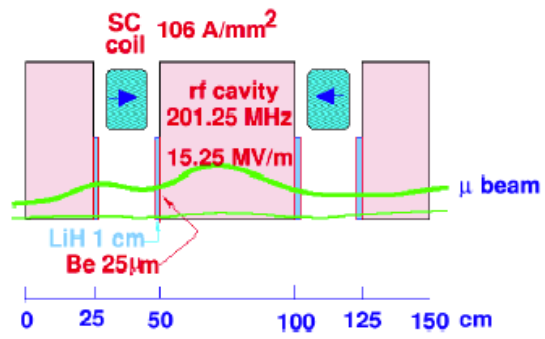

Figure 2. New cooling channel layout; absorber-RF windows are $\mathrm{LiH}$ sandwiched between Be disks. 
to accelerate the muon beam. Because these work best at higher energies, the acceleration system starts with a linac up to $1.5 \mathrm{GeV}$, then uses a "dog-bone" RLA to reach $5 \mathrm{GeV}$. Thereafter, two FFAGs accelerate the beam from 5-10 GeV and from 10-20 $\mathrm{GeV}$, respectively. A schematic of the updated acceleration system is shown in Fig. 3. We have looked into the design of the combined-function superconducting magnets required for the FFAG rings and find them to be feasible [3].

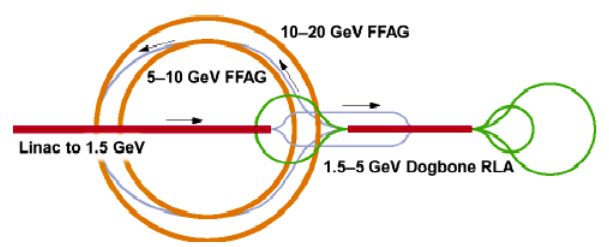

Figure 3. Schematic of Study IIa acceleration system.

\subsection{Cost Improvements}

Our estimate of the Study IIa hardware costs was obtained by scaling the cost estimates from Study II, rather than by doing a full-fledged estimate. Compared with Study II, we find that the cost in Study IIa is lower by about one-third. If we assume that the Neutrino Factory is built as an upgrade of a Superbeam facility, so that the proton driver and target complex already exist, the hardware cost of the facility is below $\$ 1 \mathrm{~B}$.

\section{ISSUES TO STUDY DURING THE ISS}

First, we must develop baseline specifications for the facility. These include: the required proton energy and intensity; detector parameters (size, baseline, technology); muon energy; need for both signs simultaneously. We also need to assess the relative costs of proton drivers for selected energies (say, 4, 20, and $50 \mathrm{GeV}$ ) and bunch lengths (say, 1 and $3 \mathrm{~ns}$ ). Another issue is to assess the practical accelerating gradient, and cost per $\mathrm{GeV}$, at several frequencies (e.g., 5, 88, and $201 \mathrm{MHz}$ ).

Since the acceleration system is costly, we need to make performance and cost comparisons of alternative acceleration systems (linacs, FFAGs, RLAs) for several values of the acceptance. This is input to a further examination of the trade-off between the amount of cooling and acceleration system acceptance. Storage ring issues also require a more careful study. Issues include the impact of multiple baselines, the need to alternate multiple signs between detectors, and the definition of required instrumentation. The question of how to "migrate" from a Superbeam facility to a Neutrino Factory also merits study, including a comparison of horn and solenoid capture systems.

To succeed in the ISS, we must develop tools for end-to-end simulations of the facility concepts. This is nontrivial, as correlations in the beam and details of the particle distribution can have significant effects on transmission at the subsystem interfaces.

The goal of examining the possibilities fairly and choosing the best ones requires buy-in from all groups, and this can only be achieved if there is a common understanding of all aspects of the design. Trade-off and costing studies will thus involve proponents from all competing systems. Fostering this "mixing" will be a task of the ISS leadership.

\section{SUMMARY}

The challenge of future "global" Neutrino Factory Studies is to try to reach consensus on a single optimized scheme. If we succeed in doing this ourselves, without the need for "referees" to choose for us, we will truly have accomplished a lot as an international community. Even if we do not quite succeed in selecting a single design, whatever convergence we achieve will improve the chances of having a future Neutrino Factory facility.

It is worth noting here that developing an optimal design requires an adequately-funded accelerator $\mathrm{R} \& \mathrm{D}$ program. It is crucial that we continue to articulate this need and define its critical elements.

\section{REFERENCES}

[1] N. Holtkamp, D. Finley (eds.), A Feasibility Study of a Neutrino Factory Based on a Muon Storage Ring, August 2000; see http://www.fnal.gov/projects/muon_collider/nu/ study/report/machine_report/.

[2] S. Ozaki, R. Palmer, M. S. Zisman, J. Gallardo (eds.), Feasibility Study II of a Muon-Based Neutrino Source, BNL-52623, June 2001; see http://www.cap.bnl.gov/mumu/studyii/final dra ft/The-Report.pdf.

[3] C. Albright, et al., Neutrino Factory and Beta Beam Experiments and Development, BNL72369-2004, FNAL-TM-2259, LBNL-55478; http://www.aps.org/neutrino/loader.cfm?url=/co mmonspot/security/getfile.cfm\&PageID=58766. 